\title{
La Pliometría en el Voleibol Femenino. Revisión Sistemática Plyometric Training in Female Volleyball Players. Systematic Review
}

*Alejandro Martínez-Rodríguez, **Jaime Mira-Alcaraz, *Bernardo J. Cuestas-Calero, *JoséAntonio Pérez-Turpín, *Pedro E. Alcaraz

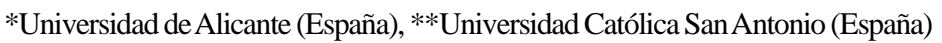

Resumen. El voleibol femenino se practica en todo el mundo a nivel profesional y amateur. Los saltos verticales son una parte fundamental para el desarrollo de este deporte, por lo que el entrenamiento pliométrico puede ser eficaz para un aumento del rendimiento. El propósito de estudio fue describir cuáles han sido los métodos y programas de entrenamiento pliométrico en mujeres que practican voleibol, así como el resultado obtenido en función de estos. El proceso de búsqueda en las bases de datos se llevó a cabo desde marzo hasta Junio de 2016. Las bases de datos consultadas fueron Pubmed, Web of Science, Cochrane y SPORTDiscus. La revisión sistemática fue realizada bajo las recomendaciones de la declaración PRISMA. La calidad metodológica de los estudios fue evaluada mediante la Escala PEDro. Como resultado, se obtuvieron un total de 205 artículos tras eliminar aquellos que estaban por duplicado. Finalmente 7 artículos fueron seleccionados. Los resultados principales fueron que aquellos estudios que utilizan deportistas amateurs obtienen mejores resultados que los realizados con deportistas profesionales cuando se realizan programas de entrenamiento pliométrico. También se indica que un período de entrenamiento pliométrico de baja intensidad puede prevenir una disminución en la altura del salto vertical; así como es necesario programar la pretemporada para evitar pérdida en la capacidad de salto. Todo ello parece indicar, como conclusión, que es necesario una disminución del volumen y un aumento de la intensidad en el entrenamiento pliométrico.

Palabras clave: salto, entrenamiento, mujeres, rendimiento, pliometría.

Abstract. Female volleyball is practiced all over the world at a professional and amateur level. Vertical jumps are a fundamental part of the development of this sport. In this sense, plyometric training can be effective for increased performance. The purpose of the study was to describe the methods and programs of plyometric training in women volleyball players, as well as the results obtained from them. The following electronic databases were searched March to June 2016. Electronic databases consulted were Pubmed, Web of Science, Cochrane and SPORTDiscus. This systematic review was performed following PRISMA statement. PEDro scale was used to evaluated the methodological quality of the included studies. As total of 205 articles were obtained after remove duplicates. Finally 7 articles were selected. The main results were that those studies using amateur athletes obtain better results than those performed with professional athletes when performing plyometric training programs. It is also indicated that a period of low intensity plyometric training could prevent a decrease in vertical jump height; As well as it is necessary to schedule the preseason to avoid loss in the ability to jump. All this seems to indicate, in conclusion, that a decrease in volume and an increase in intensity in the training of plyometric training is necessary. Key words: jump, training, women, performance, plyometric exercise.

\section{Introducción}

Elvoleibol es una disciplina deportiva de carácter explosivo donde se realizan acciones acíclicas que precisan una gran capacidad de reacción por parte del jugador y una rápida velocidad de ejecución (Marques, Tillaar, Gabbett, Reis \& Gonzalez-Badillo, 2009; Vassil \& Bazanov, 2012). En este sentido, los saltos verticales (SV) son requeridos frecuentemente durante los entrenamientos y partidos, en acciones tanto defensivas como ofensivas. Asimismo, la altura del salto en voleibol, está directamente relacionada con la velocidad de aproximación y la capacidad de controlar el centro de masas del cuerpo para transmitir esa fuerza horizontal elástica acumulada, en fuerza vertical (Ficklin, Lund \& Schipper, 2014).

En la $1^{\text {a }}$ División de voleibol de la mayor liga universitaria de Estados Unidos, la National Collegiate Athletic Association (NCAA), se realizan una media de 45 acciones que implican SV por jugadora y por partido (Conejero, Claver, Fernández-Echeverría, Gil-Arias \& Perla, 2017; Tillman, Hass, Brunt, Bennet, Jumping \& Landing, 2004).Un programa que mejore el SV puede considerarse como uno de los objetivos de la temporada y llevarse a cabo de forma específica en un momento concreto de esta. Las diferencias en cuanto a la composición corporal o las distintas respuestas fisiológicas al ejercicio físico entre sexos hacen necesaria una distinción en las planificaciones deportivas (Cabral, B.G, Cabral, S.A., Medeiros, Alcatara \& Dantas, 2013; Koley, Singh \& Singh-Sandhu, 2010; Li, 2016). Sin embargo, el voleibol es uno de los deportes donde existe mayor paridad entre las competiciones femeninas y masculinas (Ziv \& Lidor, 2010).

El entrenamiento pliométrico (EP) es una parte fundamental en el rendimiento deportivo de las jugadoras de voleibol (Fatouros, Jamurtas, Leontsini, Taxildaris, Agelouisis \& Kostopoulus, 2000; García-Asencio, Sánchez-Moreno \& González-Badillo, 2016; Wilson, Murphy \& Giorgi,

Fecha recepción: 02-01-17. Fecha de aceptación: 10-03-17 Alejandro Martínez-Rodríguez amartinezrodriguez@ua.es
1993). Este se relaciona con la fuerza reactiva y el ciclo de estiramiento acortamiento (CEA) (Malisoux, Francaux, Nielens \& Theisen, 2005). Los métodos más adecuados para mejorar son saltos verticales, horizontales y saltos en profundidad o drop jump (DJ) (Byrne, D.J., Browne, Byrne, P.J. \& Richardson, 2017). Cabe incidir, que a pesar de que la forma aceptada y extendida de medir la potencia en miembros inferiores es el test de salto CMJ (Bosco, Mognoni \& Luhtanen, 1983) por ser extrapolable a cualquier estudio y por su sencillez de ejecución, no se puede dejar de lado importancia de la carrera de aproximación previa al salto (Newton, Rogers, Volek, Häkkinen \& Kraemer, 2006) ni el salto Abalakov (ABK) por el movimiento que ejercen los brazos durante el salto (Battaglia, Paoli, Bellafiore, Bianco \& Palma, 2014; Flores, Araya, Gúzman \& Montecinos, 2015).

Sin embargo, esta relación con el gesto real del salto en voleibol y debido a la dificultad de repetir las condiciones de los estudios con estos saltos, en los cuales no sólo influye la capacidad de generar fuerza, sino la técnica de cada jugadora, se acepta la relación entre el CMJ y los distintos saltos en voleibol, como la mejor forma de medir los logros obtenidos del EP (Ziv \& Lidor, 2010). Para mejorar la efectividad de un

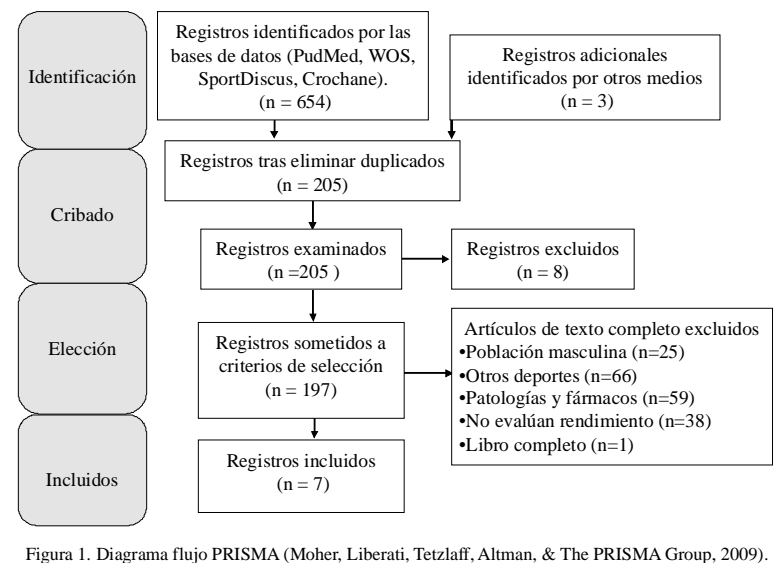

Figura 1. Diagrama flujo PRISMA (Moher, Liberati, Tetzlaff, Altman, \& The PRISMA Group, 2009). 
equipo de voleibol, las jugadoras no sólo necesitan dominar tareas técnico tácticas específicas, también deben exhibir una buena habilidad en el SV para conseguir ventaja ante la oposición del equipo rival (Pereira, Costa, Santos, Figueiredo \& João, 2015).

Por todo ello, los objetivos del presente estudio fue estudiar las diferencias en los métodos y programas de EP en jugadoras de voleibol femenino.

\section{Método}

\section{Estrategia de búsqueda}

La presente revisión sistemática fue realizada de acuerdo con las recomendaciones de la declaración PRISMA(Urútia \& Bonfill, 2010). La identificación de los artículos publicados relacionados con el EP en el voleibol femenino fue realizada a través de una búsqueda extensiva de la literatura en inglés y castellano, sin restricción de años (hasta junio de 2016) por duplicado (A.M, J.M). Las bases de datos empleadas fueron: PubMed, SPORT Discus, ISI Web of Knowledge y Cochrane. Se concretaron los descriptores de búsqueda de los Medical Subject Headings (MeSH) que fueron "plyometric exercise», «training», «athletes», «female», «volleyball». Como ejemplo de búsqueda en pubmed se presenta: [Plyometric exercise, volleyball and female] (pliometría, voleibol y mujer); [Plyometric exercise, athlete and female] (pliometría, atleta y mujer); [Plyometric training, volleyball and female] (entrenamiento pliométrico, voleibol y mujer); [Plyometric training, athlete and female] (entrenamiento pliométrico, atleta y mujer); [Plyometrics, volleyball and female] (ejercicio pliométrico, voleibol y mujer); [Plyometrics, athlete and female] (ejercicio pliométrico, atleta y mujer).

\section{Criterios de inclusión y exclusión}

La selección de los programas de EP que finalmente se incluyeron en el presente trabajo se realizó a través de la aplicación de una serie de criterios de inclusión, definidos en función de los propósitos de este trabajo y que habían de ser cumplidos en su totalidad por los estudios previamente identificados. Los criterios de inclusión fueron los siguientes: 1) Intervenciones centradas en la población femenina, entendiéndose por este colectivo aquellas chicas y mujeres de cualquier edad; 2) Dichas intervenciones tuvieron que ser llevadas a cabo específicamente con jugadoras de voleibol; 3) Personas sanas, es decir, que no padecen problemas médicos específicos; 3) Necesidad de presentar un componente de la intervención dirigido concretamente a la mejora de la altura del SV a través del EP; 4) Diseño experimental.

Los criterios de exclusión abarcaban las siguientes limitaciones: 1) Relacionados con otros deportes diferentes al voleibol; 2) Estudios que realizaban sus intervenciones en hombres; 3) Presencia de patologías y/ o suplementos y que puedan tratar la importancia del EP en sujetos no

Tabla 2.

Descripción de las características de los estudios incluidos.

\begin{tabular}{|c|c|c|c|c|c|c|c|c|c|c|c|}
\hline Estudio & & intervenc & ción & & & & iables & & & & Herramientas \\
\hline $\begin{array}{c}\text { Primer } \\
\text { autor, año }\end{array}$ & $\begin{array}{c}\text { Duración } \\
\text { (Semanas) }\end{array}$ & $\begin{array}{l}\text { Sesiones } \\
\text { (d/s) }\end{array}$ & Ejercicios & $\mathrm{SJ}(\mathrm{cm})$ & CMJ (cm) & ABK $(\mathrm{cm})$ & $\mathrm{VJ}(\mathrm{cm})$ & AVJ(cm) & $\begin{array}{c}\text { DJ-30 } \\
\text { (cm) }\end{array}$ & $\begin{array}{c}\text { DJ-60 } \\
(\mathrm{cm})\end{array}$ & \\
\hline $\begin{array}{l}\text { Arenas, } \\
2009 .\end{array}$ & 6 & 2 & COMB & $\begin{array}{l}\text { Pre: } 23,5 \pm 2,2 \\
\text { Post: } 26,0 \pm 2,4\end{array}$ & 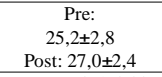 & & & & & & Plataforma de contacto. \\
\hline $\begin{array}{l}\text { Flores et al. } \\
2015\end{array}$ & 7 & N/A & COMB & $\begin{array}{c}\text { TV. Pre: } 0,5 \pm 0,02 \\
\text { TV. Post: } 0,5 \pm 0,03 \\
\text { H. Pre: } 0,3 \pm 0,03 \\
\text { H. Post: } 0,4 \pm 0,04\end{array}$ & $\begin{array}{c}\text { TV. Pre: } 0,5 \pm 0,02 \\
\text { TV. Post: } 0,6 \pm 0,03 \\
\text { H. Pre: } 0,3 \pm 0,03 \\
\text { H. Post: } 0,4 \pm 0,04\end{array}$ & $\begin{array}{c}\text { TV. Pre: } 0,6 \pm 0,02 \\
\text { TV. Post: } 0,6 \pm 0,03 \\
\text { H. Pre: } 0,4 \pm 0,03 \\
\text { H. Post: } 0,4 \pm 0,03\end{array}$ & & & & & $\begin{array}{l}\text { Analizador de marcha y } \\
\text { salto (Artoficio modelo } \\
\text { AMS-1) y estadiómetro. }\end{array}$ \\
\hline $\begin{array}{l}\text { Fry et al. } \\
1991 \\
\end{array}$ & 12 & 3 & COMB & & & & $\begin{array}{r}\text { Pre: } 44,7 \pm 5,7 \\
\text { Post: } 48,0 \pm 4,2 \\
\end{array}$ & $\begin{array}{l}\text { Pre: } 47,6 \pm 5,0 \\
\text { Post: } 51,8 \pm 5,6\end{array}$ & & & $\begin{array}{c}\text { Marca más alta en la pared } \\
\text { (mano) }\end{array}$ \\
\hline $\begin{array}{l}\text { Lehnert et } \\
\text { al. } 2009\end{array}$ & 8 & 2 & COMB & & & & $\begin{array}{c}\text { Pre: } 29,5 \pm 3,8 \\
\text { Dur: } 30,45 \pm 3,53 \\
\text { Post: } 32,09 \pm 4,08 \\
\text { Post-3: } 1,18 \pm 5,03 \\
\text { Post-6: } 33,54 \pm 4,08\end{array}$ & $\begin{array}{c}\text { Pre: } 38,33 \pm 5,46 \\
\text { Dur: } 39,63 \pm 6,56 \\
\text { Post: } 42,63 \pm 6,28 \\
\text { Post-3: } 41,63 \pm 6,21 \\
\text { Post-6: } 43,27 \pm 5,98 \\
\end{array}$ & & & $\begin{array}{l}\text { Marca más alta en la pared } \\
\text { (mano) }\end{array}$ \\
\hline $\begin{array}{l}\text { Marques et } \\
\text { al. } 2008\end{array}$ & 12 & 2 & CMJ & & $\begin{array}{l}\text { Pre: } 34,22 \pm 5,90 \\
\text { Post: } 35,56 \pm 6,28\end{array}$ & & & & & & $\begin{array}{l}\text { Plataforma de fuerza (Ergo } \\
\text { Jump Digitime 1000) }\end{array}$ \\
\hline $\begin{array}{l}\text { Newton et } \\
\text { al. } 2006\end{array}$ & 4 & 2 & $\begin{array}{l}\text { Sentadilla salto } \\
\text { en máquina } \\
\text { Smith }\end{array}$ & $\begin{array}{l}\text { Pre: } 38 \pm 3 \\
\text { Dur: } 36 \pm 2 \\
\text { Post: } 37 \pm 3\end{array}$ & $\begin{array}{l}\text { Pre: } 40 \pm 4 \\
\text { Dur: } 39 \pm 3 \text {, } \\
\text { Post: } 40 \pm 3\end{array}$ & & & & $\begin{array}{l}\text { Pre: } 40 \pm 5 \text {, } \\
\text { Dur: } 39 \pm 3 \text {, } \\
\text { Post: } 41 \pm 4\end{array}$ & $\begin{array}{l}\text { Pre: } 38 \pm 4 \text {, } \\
\text { Dur: } 39 \pm 4 \text {, } \\
\text { Post: } 38 \pm 3\end{array}$ & $\begin{array}{l}\text { Plataforma de fuerza } \\
\text { (Quattro Jump Kistler) }\end{array}$ \\
\hline $\begin{array}{l}\text { ereira et al. } \\
2015\end{array}$ & 8 & 2 & COMB & & & $\begin{array}{l}\text { Pre: } 25 \pm 3,7 / 26,9 \pm 4,5 \\
\text { Post: } 25,8 \pm 3,7 / 32,3 \pm 9 \\
\end{array}$ & & & & & $\begin{array}{l}\text { Plataforma de fuerza (Ergo } \\
\text { Jump Digitime 1000) }\end{array}$ \\
\hline
\end{tabular}

2015 Post: $25,8 \pm 3,7 / 32,3 \pm 9 \quad$ Jump Digitime 1000$)$ )

vertical), AVJ (salto vertical con aproximación), DJ-30, (drop jump desde 30 centímetros), DJ-60 (drop jump desde 60 centímetros).cm (centímetros), N/A (no aparece), COMB (combinación de ejercicios), TV ( tiempo de vuelo), $H$ (altura), Pre (pretest), Dur (test a mitad de intervención), Post (postest), Post-3 (postest 3 semanas después de la intervención), Post- 6 (postest 6 semanas después de la intervención). sanos; 4) Aquellos estudios observacionales que no aplicaban ningún tipo de entrenamiento; 5) Estudios que no evalúan parámetros de rendimiento deportivo.

La evaluación de la calidad metodológica de los artículos seleccionados fue elaborada mediante la escala PEDro(Maher, Sherrigton, Herbert, Moseley \& Elkins, 2003).

\section{Resultados}

El diagrama de flujo (figura 1), muestra el proceso de identificación, cribado, elegibilidad e inclusión de los artículos de la presente revisión sistemática. De un total de 654 artículos identificados, más el registro de 3 artículos adicionales, el cribado tras eliminar los artículos duplicados tuvo 205 artículos. Se descartaron en primera instancia aquellos documentos que hacían referencia a patentes o temas de conferencias ( $\mathrm{n}=8$ ). En segunda, fueron excluidos los estudios que no cumplían con los criterios de inclusión referidos anteriormente en el apartado de metodología (n=189). Finalmente, 7 artículos fueron seleccionados (Arenas, 2009; Flores et al., 2015; Fry, Kraemer, Weseman, Conroy, Gordon, Hoffman \& Maresh, 1991; Lehnert, Lamrová \& Elfmark, 2009; Marques, Tillaar, Vescovi \& Gonzalez-Badillo, 2008; Pereira et al., 2015; Ziv \& Lidor, 2010).

\section{Tamaño de la muestra}

La tabla 1 presenta los datos referentes a las características de las jugadoras participantes en cada uno de los estudios seleccionados. Asimismo, la tabla 2 hace referencia a las especificaciones de cada uno delos estudios, como el número de sesiones, la duración, o ejercicios del entrenamiento realizado. Como se puede apreciar en las tablas 1 y $2 \mathrm{el}$ número de jugadoras que realizan el entrenamiento en cada estudio es diferente. Con una media de 12 deportistas, se encuentran cuatro artículos por debajo de esta media (Arenas, 2009; Flores et al., 2015; Lehnert et al., 2009; Marques et al., 2008) y tres las investigaciones que la superan (Fry et al., 1991; Newton et al., 2006; Pereira et al., 2015).

\section{Edad y nivel de la muestra}

En lo que refiere a la edad de las jugadoras, existe una gran variedad. Por bloques, se puede agrupar por un lado aquellos estudios que utilizan deportistas amateurs y juveniles con edades comprendidas entre

Tabla 1.

\begin{tabular}{|c|c|c|c|c|c|}
\hline \multirow{2}{*}{$\begin{array}{c}\text { Estudio } \\
\text { Primer autor }\end{array}$} & \multicolumn{5}{|c|}{ Participantes } \\
\hline & $\mathbf{n}$ & Sexo & Edad (años) & Categoría & Nivel \\
\hline Arenas, 2009. & 6 & Mujeres & $15,5 \pm 1,5$ & Juvenil & Amateur \\
\hline Flores et al. 2015 & 9 & Mujeres & $15,4 \pm 0,7$ & Juvenil & Amateur \\
\hline Fry et al. 1991 & 14 & Mujeres & $19,7 \pm 0,5 / 19,5 \pm 0,8$ & Senior & NCAA División I \\
\hline Lehnert et al. 2009 & 11 & Mujeres & $14,8 \pm 0,9$ & Juvenil & Amateur \\
\hline Marques et al. 2008 & 10 & Mujeres & $25,3 \pm 1,3$ & Senior & Profesional \\
\hline Newton et al. 2006 & 14 & Mujeres & $20,0 \pm 1,2$ & Senior & NCAA División I \\
\hline Pereira et al. 2015 & 20 & Mujeres & $13,8 \pm 0,4 / 14,0 \pm 0,0$ & Juvenil & Amateur \\
\hline
\end{tabular}

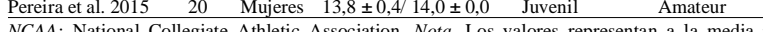
NCAA: National Collegiate Athletic Association. Nota. Los valores representan a la media \pm
desviación estándar. 
13,8 \pm 0,4 y 15,4 \pm 0,7 años (Flores et al., 2015; Lehnert et al., 2009; Pereira et al., 2015) y por el otro lado aquellos que utilizan una muestra de jugadoras de voleibol profesionales de edades comprendidas entre $19,5 \pm 0,8$ y 25,3 $\pm 1,3$ años y con el desarrollo de su actividad en la NCAA División I, en la $1^{\text {a }}$ división portuguesa en incluso selección nacional (Fry et al., 1991; Marques et al., 2008; Newton et al., 2006).

\section{Métodos de evaluación y variables analizadas}

En los estudios seleccionados aparecieron diferentes métodos de evaluación, además de llevarse a cabo mediante distintas herramientas. En relación a la evaluación de la potencia en los miembros inferiores, la mayoría de los autores emplearon el CMJ. Sin embargo, también se llevaron a cabo otros tipos de SV para la realización de los test de evaluación del salto en jugadoras de voleibol (Arenas, 2009; Flores et al.,2015; Fry et al., 1991; Lehnert et al., 2009; Newton et al., 2006; Ziv \& Lidor, 2010).

Los trabajos revisados utilizaron estadímetros, plataformas de contacto (Arenas, 2009; Flores et al., 2015; Newton et al., 2006; Pereira et al., 2015), marca más alta en la pared (Fry et al., 1991; Lehnert et al., 2009) y tiempo de vuelo y altura (Flores et al., 2015). El resto de trabajos utilizaron como variable a comparar la altura de SV y en algunos casos se estimaron, pero no se midieron (Arenas, 2009; Marques et al., 2008; Pereira et al., 2015).

\section{Duración de los programas de EP}

A excepción de Fry et al. (1991) el cual realizó un programa de entrenamiento dos semanas después de acabar la temporada, cuando las jugadoras no tienen partidos de competición. El resto de artículos ubicaron sus EPa lo largo de la temporada regular. Sin embargo estos programas son diferentes entre sí. En ocasiones hasta se triplica la duración. De menor a mayor duración se muestran EP de 4 semanas (Newton et al., 2006), 6 semanas (Arenas, 2009), 7 semanas (Flores et al., 2015), 8 semanas (Lehnert et al., 2009; Pereira et al., 2015) y 12 semanas (Fry et al., 1991; Marques et al., 2008).

\section{Volumen e intensidad de los programas de EP}

Por un lado se presentaron los artículos que pretenden mejorar la altura del SV con cargas altas (Arenas, 2009; Flores et al., 2015; Lehnert et al., 2009; Pereira et al., 2015) y por otro lado, aquellos que iban en la línea de reducir la intensidad para lograr una supercompensación para afrontar determinados momentos de la temporada ( Fry et al., 1991; Marques et al., 2008; Newton et al., 2006). En todos los artículos el EP se combinó con diferentes entrenamientos de fuerza, resistencia, técnica y/o táctica.

\begin{tabular}{|c|c|c|}
\hline Autores & Media de saltos por sesión & Número de saltos totales \\
\hline Arenas, 2009 & 156 & 1720 \\
\hline Flores et al. 2015 & 131 & 915 \\
\hline Pereira et al. 2015 & 149 & 2390 \\
\hline Lehnert et al. 2009 & 123 & 1964 \\
\hline Marques et al. 2008 & 59 & 1245 \\
\hline Newton et al. 2006 & 14 & 108 \\
\hline Fry et al. 1991 & 115 & 4140 \\
\hline
\end{tabular}

Como se pudo observar en la tabla 3 , aquellos estudios que llevaron a cabo un programa de EP con cargas altas (Arenas, 2009; Flores et al., 2015; Lehnert et al., 2009; Pereira et al., 2015) tuvieron una media de saltos por sesión similar $140 \pm 17$. Por el contrario, los autores que trabajaron con cargas bajas (Fry et al., 1991; Marques et al., 2008; Newton et al., 2006) tuvieron una media de saltos con una desviación típica de $63 \pm 52$ saltos. Tanto Marques et al. (2008), como Newton et al. (2006), plantearon un trabajo basado en un aumento de la intensidad, en el que emplearon cargas óptimas. Fry et al. (1991) propusieron un EP de baja intensidad y sin carga externa adicional. Asimismo, en la columna del número de saltos totales de la tabla 3, se observó que Fry et al. (1991) plantearon un EP de «baja intensidad», siendo el volumen total de saltos que realiza cada jugadora es el mayor de todos los estudios. En este sentido, junto al estudio de Marques et al. (2008), son los autores que programaron 12 semanas de EP, además también plantearon la realización de 3 sesiones semanales.

\section{Efectos del EP}

Todos los programas de EP propuestos por los diferentes autores (tabla 2), parecen contribuir en la mejora de la altura del SV en jugadoras de voleibol. Arenas (2009), obtuvo unas mejoras en SJ y CMJ del $10,6 \%$ y el 7,1\% respectivamente realizando un entrenamiento muy variado en el cual no se incluyó carga externa, pero sí una gran variedad de saltos tanto verticales como horizontales, ejercicios de coordinación y relacionados con los gestos de remate y bloqueo en voleibol. Flores et al. (2015) propusieron un programa de EP con multisaltos horizontales y verticales, saltos en profundidad y DJ, que les permitió obtener una mejora en la altura del salto tanto en SJ (33,3\%) como en CMJ (33,3\%) pero no en $\mathrm{ABK}$.

Por su parte, Lehnert et al. (2009) no sólo realizaron mediciones antes y después del entrenamiento; sino que también las realizaron durante y tras acabar el programa para obtener una visión más amplia de lo que su EP puede llegar a mejorar la altura del SV. En una primera evaluación, justo a la mitad del programa, obtuvieron una mejora del 3,2\% en VJ y del 3.4\% en AVJ; al finalizar el programa (8 semanas) obtuvieron mejoras tanto en VJ, como en AVJ del 8,8\% y del 11,2\% respectivamente. Asimismo, tras 3 y 6 semanas del EP registraron mejoras en VJ del 5,7\% y 13,7\% y en AVJ del 8,5\% y 12,9\%. No obstante, también se observó que 3 semanas después del EP la altura del salto en ambos caso se ve reducida con respecto a la medición post entrenamiento, sin embargo el efecto residual del programa parece mostrar una mejora sustancial tras 6 semanas de ausencia en EP específico tanto en VJ como en AVJ.

Pereira et al. (2015) fueron los únicos autores que diferenciaron entre grupo experimental (que realiza el EP programado) y grupo control (que no realiza un entrenamiento específico). Con una batería, que a diferencia del resto incluyeron el componente de la velocidad en la realización de los ejercicios, realizando mediciones pre-post de CMJ, y que obtuvieron mejoras en ambos grupos. El grupo control mejoró tras 8 semanas de entrenamiento un 3,2\% frente a un $20,1 \%$ del grupo experimental.

Todos los estudios anteriormente comentados (Arenas, 2009; Flores et al., 2015; Lehnert et al., 2009; Pereira et al., 2015) desarrollaron su investigación con una muestra de deportistas juveniles, obteniéndose su gran porcentaje de mejora. A continuación, se muestran los estudios que utilizaron una muestra más experimentada, como el estudio de Fry et al. (1991) el cual estudió a deportistas de la NCAADivisión I Team. Estos autores dividieron a su grupo de deportistas en titulares y suplentes y tras 12 semanas de EP obtuvieron mejoras en la altura del VJ en titulares (5\%) y en suplentes (13,2\%) y también en AVJ (5,9\% en titulares y $14,9 \%$ en suplentes).

Marques et al. (2008) por su parte, propusieron un programa de EP simple formado por los mismos 3 ejercicios por sesión pero con distinta carga externa y tras 12 semanas de EP obtuvieron mejoras en CMJ del 3.9\%. Por último, Newton et al. (2006) con un único ejercicio (Sentadilla con salto en máquina Smith con carga óptima) y una media de 14 saltos por sesión, también obtuvieron mejoras en CMJ del 2,6\%, en AVJ del 5,4\% y en SJ del 2,8\%.

A modo de resumen, las mejoras en la altura de salto planteadas en

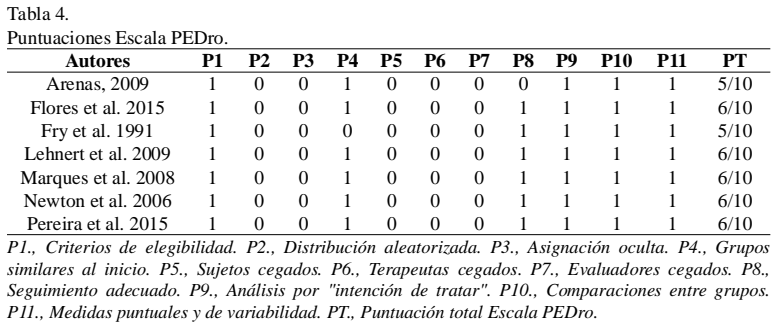


los estudios analizados oscilante entre el 2,6\% hasta $14,8 \%$ en jugadoras de élite, y desde el 7,1\% hasta el 33.3\% en deportistas amateurs.

La calidad de los artículos seleccionados, que fueron medidos mediante la Escala PEDro mostraron un promedio de 5,7 $\pm 0,5$ puntos (tabla 4).

\section{Discusión}

La presente revisión pretendía determinar la influencia de un programa de EPen jugadoras de voleibol femenino. Al igual que ocurre con otras habilidades y capacidades como, por ejemplo, la potencia en lanzamiento de faltas en fútbol o en el lanzamiento del pitcher en béisbol. No está claro que la habilidad en el SV esté directamente relacionada con el éxito en los partidos de voleibol, aunque es importante mejorarla en jugadoras debido a que es un elemento básico en el desarrollo del juego (Ziv \& Lidor, 2010). La potencia explosiva en los músculos de las piernas es una parte importante de la mejora del desarrollo del SV (Adams, O'Shea J.P., O'Shea K. L. \& Climstein, 1992), y el último meta análisis que relaciona la altura del SV con el EP sugiere que el EP puede aumentar la altura del SV en $>7 \%$ (i.e., 3,90cm) (De Villarreal, Kellis, Kraemer \& Izquierdo, 2009).

Como se ha observado en los resultados, la media de sujetos de las investigaciones se puede considerar aceptable, en conjunto, el cómputo global de los estudios analizados es escaso ( $\mathrm{n}=7$ ), y si además tenemos en cuenta la disparidad en las edades de la muestra, separándola en dos grupos (juveniles y sénior) la cifra total aún es menor. En la misma línea, algunos de los estudios analizados no utilizan sujetos muy entrenados y por lo tanto los resultados son favorables por el simple hecho de la mejora general de la condición física. La duda que nos ocupa es si realmente existen diferencias significativas tras el EP en una población que ya domina o tiene una alta competencia en SV. Izquierdo, Häkkinen, Gonzalez-Badillo, Ibañez \& Gorostiaga (2002), sugieren que la diferencias en la sección transversal del músculo, la distribución del tipo de fibras y los patrones neurofisiológicos, así como la formación específica en un deporte durante los años, puede contribuir a explicar las diferencias en las magnitudes expuestas entre atletas de diferentes deportes. Por esta razón se sugiere que un entrenamiento específico alrededor de 3 años, produce adaptaciones en SV en comparación con individuos de la misma edad que no lo hayan realizado (Battaglia et al., 2014).

El entrenamiento balístico normalmente hace referencia al relacionado con manifestaciones activas de la fuerza como la fuerza máxima dinámica y la fuerza explosiva, sin incluir ciclo de acortamiento estiramiento (e.g. sentadilla máxima o squat jump) (López-Calbet, Chavaren \& Dorado, 1995). En los estudios analizados, ambos términos estarán englobados bajo las siglas EP y están relacionados con el entrenamiento que pretende incrementar la potencia muscular de los miembros inferiores. En este sentido, destacar que diversos autores indican que el aumento de la fuerza explosiva de las piernas guarda una relación directa con la capacidad para saltar más alto (Adams et al.,1992).

A partir de los resultados de los estudios seleccionados, parece recomendable realizar mediciones de las variables de salto, una vez que haya pasado un tiempo tras finalizar los programas de EP (Lehnert et al., 2009). Los resultados de Lehnert et al. (2009) plantearon que el análisis de los datos obtenidos muestra un pequeño incremento entre la $1^{\mathrm{a}}$ y la $4^{\mathrm{a}}$ semana, lo que puede estar relacionado con la baja intensidad de los ejercicios realizados en la primera parte del programa, teniendo en cuenta la carencia de rigurosidad del protocolo y el nivel de los deportistas.

En lo referente a los protocolos de medición y al tipo de variables analizadas, se precisa necesario unificar más los criterios, tanto la altura del salto como el tiempo de vuelo son variables válidas para llevar a cabo un control de un equipo (García-López et al., 2005). Sin embargo, se debe intentar obtener estas variables de manera directa, sin estimaciones calculadas que puedan acumular errores en el posterior análisis de los resultados (Moon et al., 2009). En los estudios seleccionados, la forma de realizar estas mediciones fue muy variable, en función de aquello que se pretenda medir. Para posteriores trabajos sería estandarizar las herra- mientas empleadas en función de la variable de estudio. En este sentido, destacar que el uso de diferentes herramientas o aparatos de medida puede conllevar diferentes valores de medición para un mismo salto, debido a los errores de cada una de estas herramientas (García-López, Peleteiro, Rodriguez-Marroyo, Morante, Herrero \& Villa, 2005), incluso, distintas marcas y modelos de plataforma de contacto pueden llegar a registrar resultados con una diferencia del 2.9\% (Aragón, 2000).

En relación a los saltos empleados, como variables de estudio, parece ser que la mayoría de los autores utilizan el CMJ para medir el salto en los deportistas. Esto puede deberse a su sencillez de aprendizaje y ejecución, además de la capacidad de ser extrapolable a otros estudios (Bosco et al., 1983; De Villarreal et al., 2009; Pereira et al., 2015; Robinson, Devor, Merrick \& Buckworth, 2004; Wilson et al., 1993). No obstante, otros tipos de salto como el VJ, ABK, AVJ, SJ o DJ, no tienen por qué asociarse a estudios de menor calidad si se realiza un buen protocolo en el desarrollo del test. Además, son varios los autores que opinan que la relación entre el entrenamiento y el juego real ha de ser lo más próxima posible (Gimeno, Buceta \& Perez-Llantada, 2007), por tanto el salto AVJ no sólo no se considera no válido, sino que puede ser el que mayor transferencia tenga en el juego del voleibol.

En cuanto a la duración de los distintos diseños se mostró una gran diversidad en ellos. Newton et al. (2006) realizan un programa excesivamente corto en comparación con el resto, sin embargo esto es debido a que estos autores pretendieron desarrollar, más que un complejo EP, un programa reducido, con cargas óptimas y su eficacia en el SV; en realidad, estos autores solamente propusieron un ejercicio de sentadilla con salto en máquina con distintas combinaciones de series y repeticiones como EP. En base a la literatura relacionada con la pliometría y el voleibol (Asadi, Arazi, Young \& Saez; De Villarreal, 2016), no se entiende un programa de entrenamiento que busque el rendimiento en este deporte que omita los ejercicios balísticos o de pliometría en todo su espectro. Desde el inicio de la pretemporada hasta el final de la temporada, el deporte en sí mismo contiene movimientos que implican el CEA en un porcentaje muy elevado de sus acciones (Fatouroux et al., 2000; Malisoux et al., 2005). Por tanto, un programa de EP para jugadoras de voleibol debería durar tanto como dure la temporada.

En relación con lo comentado anteriormente, y analizados los programas de entrenamiento de voleibol, parece que el problema radica en si se contabiliza o no ese entrenamiento en cuanto a volumen e intensidad. Esto es, en un primer momento, realizar un análisis del número de SV que se realizan en un partido, y a posteriori incrementar o disminuir las cargas externas en función de las necesidades de cada jugadora. Al respecto, indicar que aunque la cantidad media de saltos por sesión no seas excesivamente alta (Ziv \& Lidor, 2010), la cantidad de entrenamientos se traduce en un volumen de carga alta.

Como se ha mencionado en los resultados, todos los artículos revisados obtuvieron mejoras en las variables medidas ya sea tiempo de vuelo como altura de salto. Estos resultados van en la línea de otros trabajos relacionados con la pliometría en otros deportes (Asadi et al., 2016; Marqueste, Messan, Hug, Laurin, Dousset, Grelot \& Decherchi, 2003) y con el EP en jugadores de voleibol (Maffiuletti, Dugnani, Folz, Di Pierno \& Mauro, 2002; Pereira, Almeida, Ugrinowitsch, Fowler \& Kokubun, 2008). Dicho esto, se observa que durante el entrenamiento tradicional no sólo no existe un aumento en la altura de los saltos, sino que se produce un descenso de esta (Maffiuletti et al., 2002, Marques et al., 2008; Newton et al, 2006; Marques \& Gonzalez-Badillo, 2005), cuando se trata de jugadoras sénior de alto nivel. Sin embargo, tras la aplicación del programa, las jugadoras recuperan los valores de salto del inicio de la temporada, y si bien es cierto que no se produjo un aumento significativo en ninguno de los SV testados, conseguir llegar al final de la temporada con unos valores similares a los iniciales puede ser muy interesante para entrenadores y atletas. Estos estudio contradice los resultados obtenidos por Häkikinen (1993) donde mostraban que cinco semanas y media sin entrenamiento de alta intensidad podría ser demasiado tiempo y conllevaba una pérdida de la capacidad de salto.

Dentro de las limitaciones En base a la revisión realizada de los estudios seleccionados para este trabajo, se encuentran las siguientes 
limitaciones: a) Empleo de gran cantidad de protocolos de EP: En cada uno de los 7 artículos seleccionados se especifica el tipo de EP empleado. Estos entrenamientos varían tanto en intensidad como en volumen. b) Utilización de diferentes edades y niveles deportivos de la muestra lo cual hace difícil extraer conclusiones generales. c) Variabilidad en la duración de los programas de EP y en el momento de la temporada en que fueron puestos en práctica. d) Utilización de diversos aparatos de medición, en alguno de los cuales no se especifica el modelo, así como de diferentes test y diferentes variables medidas. e) Ausencia de una escala que mida la calidad de los artículos que se aproxime en mayor medida al tipo de estudios del ámbito del rendimiento deportivo.

Implicaciones prácticas

En base a los artículos revisados se pueden sugerir las siguientes implicaciones prácticas para entrenadores de voleibol y preparadores físicos:

1) El EP presenta cierta predisposición a mejorar la altura del SV en jugadoras de voleibol y aunque algunos autores han llegado a la conclusión que el EP puede no ser efectivo si se trata de manera aislada (Chaouachi, Othman, Hammami, Drinkwater \& Behm, 2014) y son muchos los trabajos que exponen la importancia EP incluso para prevenir lesiones (Halvorson, 2011; Ismail, Ibrahim, Youssef \& El Shorbagy, 2010). Como muestran los artículos analizados, es recomendable incluir sesiones o ejercicios de pliometría en la planificación de una temporada de voleibol; el volumen y la intensidad va a depender de todos los factores que rodeen a cada equipo y a cada jugadora en cada momento.

2) Un aumento de la intensidad de la carga no siempre conlleva un aumento del rendimiento. Como se ha apreciado, la inclusión de un programa de EP mejora la altura del SV en todos los casos estudiados, sin embargo no siempre estos entrenamientos ha supuesto un aumento de la carga (Di Giminiani \& Petricola, 2016). La introducción de un programa de EP con bajas cargas, no más de 4 semanas si no se quiere caer en un descenso de la forma física (Häkikinen,1993), pueden ayudar al organismo a conseguir una mejora en la altura del salto.

3) El momento de la temporada en la que aplicar los diferentes programas de EP puede ser variable y va a depender de los deportistas con los que trabajemos. Una fluida relación entre los entrenadores, los preparadores físicos y las jugadoras es fundamental para no derivar en el sobreentrenamiento (Ziv \& Lidor, 2010). Sin esta cooperación, no se puede planificar siguiendo los principios del entrenamiento; así, en función de las lesiones, el estado de forma al iniciar la pretemporada, los minutos jugados por cada jugadora o la capacidad física de estas, entre otros factores, debemos plantear el programa de EP en la pretemporada o a lo largo de la temporada.

4) Para conseguir ser capaces de aplicar con éxito un programa de EP u otro, puede ser interesante el uso de test que nos indiquen el estado de forma de las jugadoras en cada momento de la temporada. Son varios los estudios que nos indican que la manera de entrenar con la carga óptima de entrenamiento es la mejor forma de lograr los objetivos marcados (Buchheit \& Laursen, 2013; Loturco, Ugrinowitsch, Roschel , Tricoli \& González-Badillo, 2013; Marques et al., 2008). Por ello es necesario variar la carga de entrenamiento en función de la adaptación de las deportistas a ella. Un sencillo test como el CMJ con carga externa variable en plataforma de fuerzas, nos puede delimitar con exactitud la carga óptima en cada momento y trabajar en función de esta y del momento de la temporada en el que nos encontremos.

En vista de las limitaciones halladas en esta investigación, se recomienda como futuras líneas de investigación unificar criterios a la hora de medir y evaluar el SV en jugadoras de voleibol así como aumentar el número de trabajos relacionados con el ámbito de la pliometría en el voleibol femenino.

\section{Conclusiones}

Las conclusiones de la presente revisión fueron que, de un total de 7 estudios analizados, parece ser que la edad y la categoría influyen sobre el número de saltos que realizan en las sesiones de entrenamiento. Las deportistas jóvenes o amateur realizan un mayor número de saltos por sesión que las deportistas senior o entrenadas. Dentro de los diferentes saltos empleados en la valoración de la fuerza explosiva de las deportistas, el CMJ fue el más empleado por su sencillez de ejecución. En cuanto a las diferentes herramientas de medición utilizadas la plataforma de contacto fue la más utilizada por su fiabilidad. Con respecto a las variables de medición los estudios analizados optan por medir altura o tiempo. Son muy pocos los trabajos que siguen una línea de investigación común, puesto que aunque todos los trabajos expuestos tratan la pliometría en el voleibol femenino, hay una gran diferencia entre los protocolos utilizados y dificultan la comparación de los mismos. Son necesarias más investigaciones al respecto para poder definir de forma más exhaustiva los procedimientos y desarrollo de los programas deEP.

\section{Referencias}

Adams, K. J., O’Shea, J. P., O’Shea, K. L., \& Climstein, M. (1992). The effect of six weeks of squat, plyometrics, and squat-plyometric training on power production. Journal of Applied Sport Science Research, 6(1), 34-41.

Aragón, L. F. (2000). Evaluation of Four Vertical Jump Tests: Methodology, Reliability, Validity, and Accuracy. Measurement in physical Evaluation and Exercise Science,4(4), 215-228.

Arenas-Bustamante, J.D. (2009). Influencia de un plan de seis semanas de entrenamiento pliométrico de moderada intensidad en miembros inferiores sobre el índice elástico de las jugadoras de voleibol femenino de la Institución Educativa INEM José Félix de Restrepo con edades que oscilan entre los 14 y 17 años (Informe de investigación). Universidad de Antioquia, Medellin, Colombia.

Asadi, A., Arazi, H., Young, W. B., \& Sáez de Villarreal, E. (2016). The Effects of Plyometric Training on Change-of-Direction Ability: A Meta-Analysis. International Journal of Sports Physiology and Performance, 11(5), 563-573.

Battaglia, G., Paoli, A., Bellafiore, M., Bianco, A., \& Palma, A. (2014). Influence of a sport-specific training background on vertical jumping and throwing performance in young female basketball and volleyball players. The Journal of Sports medicine and physical fitness, 54(5), 581-587.

Bosco, C., Mognoni, P., \& Luhtanen, P. (1983). Relationship between isokinetic performance and ballistic movement. European Journal of Applied physiology and occupational physiology,51(3), 357-364.

Buchheit, M., \& Laursen, P. B. (2013). High-intensity interval training, solutions to the programming puzzle: Part I: cardiopulmonary emphasis. Sports Medicine, 43(5), 313-338.

Byrne, D. J., Browne, D. T., Byrne, P.J., \& Richardson, N. (2017). The Inter-Day Reliability of Reactive Strength Index and Optimal Drop Height. The Journal of Strength and Conditioning Research,31(3), 721-726.

Cabral, B. G, Cabral, S. A., Medeiros, R. M., Alcatara, T., \& Dantas, P.M.S. (2013). Relação da maturação com a antropometria e aptidão física na iniciação desportiva. Motricidade, 9(4), 12-21.

Chaouachi, A., Othman, A. B., Hammami R., Drinkwater, E. J., \& Behm, D. G (2014). The combination of plyometric and balance training improves sprint and shuttle run performances more often than plyometric-only training with children. The Journal of Strength and Conditioning Research, 28(2), 401-412.

Conejero, M., Claver, F., Fernández-Echeverría, C., Gil-Arias, A., \& Perla, M. (2017). Toma de decisiones y rendimiento en las acciones de juego intermedias y finalistas en voleibol, en sets con diferente resultado. Retos, 31, 28-33.

De Villarreal, E. S., Kellis, E., Kraemer, W. J., \& Izquierdo, M. (2009). Determining variables of plyometric training for improving vertical jump height performance: a meta-analysis. The Journal of Strength and Conditioning Research, 23(2), 495-506.

Di Giminiani, R., \& Petricola, S. (2016). The Power Output-Drop Height Relationship to Determine the Optimal Dropping Intensity and to Monitor the Training Intervention. The Journal of Strength and Conditioning Research, 30 (1), 117-125. 
Fatouros, I. G., Jamurtas, A. Z., Leontsini, D., Taxildaris, K., Aggelousis, N., Kostopoulos, N., \& Buckenmeyer P. (2000). Evaluation of Plyometric Exercise Training, Weight Training, and theircombination on vertical jumping performance and leg Strength. Journal of Strength and Conditioning Research, 14(4), 470-476.

Ficklin, T., Lund, R., \& Schipper. M. (2014). A comparison of jump height, takeoff velocities, and blocking coverage in the swing and traditional volleyball blocking techniques. Journal of Sports Science \& Medicine, 13(1), 78-83.

Flores, A., Araya, S., Guzmán, R., \& Montecinos, R. (2015). Efecto de un programa de entrenamiento pliométrico sobre la biomecánica de salto en mujeres voleibolistas juveniles. Revista Ciencias de la Actividad Física UCM, 16(1), 37-44.

Fry, A. C., Kraemer, W. J., Weseman, C. A., Conroy, B. P., Gordon, S. E., Hoffman, J. R., \& Maresh, C. M. (1991). The effects of an offseason strength and conditioning program on starters and nonstarters in women's intercollegiate volleyball. Journal of Strength and Conditioning Research, 5(4), 174-181.

García-Asencio, C., Sánchez-Moreno, M., \& González-Badillo, J. J. (2016). Entrenamiento combinado de fuerza y ejercicios de saltos, efectos sobre el rendimiento en el salto vertical en un grupo de alto nivel de jugadores de voleibol durante una temporada completa de competición. Retos, 29, 140-143.

García-López, J., Peleteiro, J, Rodgríguez-Marroyo, J. A., Morante, J. C., Herrero, J. A., \& Villa, J. G. (2005). The validation of a new method that measures contact and flight times during vertical jump. International Journal of Sports Medicine, 26(4), 294-302.

Gimeno, F, Buceta, J. M., y Pérez-Llantada, M. C. (2007). Influencia de las variables psicológicas en el deporte de competición: evaluación mediante el cuestionario características psicológicas relacionadas con el rendimiento deportivo. Psicothema, 19(4), 667-672.

Häkkinen, K. (1993). Changes in physical fitness profile in female volleyball players during the competitive season. The Journal of Sports Medicine and Sports physical fitness, 33(3), 323-332.

Halvorson, R. (2011). Jump Training Reduces Injury Potential in Females. IDEA Fitness Journal, 8(4), 18.

Ingen-Schenau, G., Bobbert, M. F., \& Haan, A. (1997). Does elastic energy enhance worf and efficiency in the stretch-shortening cycle? Journal of Applied Biomechanics, 13(4), 389-415. Doi: http:// dx.doi.org/10.1123/jab.13.4.389.

Ismail, M. M., Ibrahim, M.M., Youssef, E. F., \& El Shorbagy, K.M. (2010). Plyometric training versus resistive exercises after acute lateral ankle sprain. Foot \& Ankle International, 31(6), 523-530.

Izquierdo, M., Häkkinen, K., Gonzalez-Badillo, J. J., Ibáñez, J., \& Gorostiaga, E. M. (2002). Effects of long-term training specificity on maximal strength and power of the upper and lower extremities in athletes from different sports. European Journal of Applied Physiology, 87(3), 264-271.

Koley, S., Singh, J., \& Singh-Sandhu, J. (2010). Anthropometric and physiological characteristics on Indian inter-university volleyball players. Journal of Human Sport and Exercise, 5(3), 389-399.

Lehnert, M., Lamrová, I., \& Elfmark, M. (2009). Changes in Speed and Strength in Female Volleyball Players During and After a Plyometric Training Program. Acta Universitatis Palackianae Olomucensis Gymnica, 39(1), 59-66.

Li, L. (2016). The Effects of Body Mass on Optimal Load for Power During Resistance Training. Sports Meddicine, 46(3), 443-445.

López-Calbet, J. A., Arteaga, R., Chavaren, J., y Dorado, C. (1995). Comportamiento mecánico del músculo durante el ciclo estiramiento-acortamiento. Factores neuromusculares. Archivos de Medicina del Deporte, 12(47), 219-223.

Loturco,I., Ugrinowitsch, C., Roschel, H., Tricoli, V., \& GonzálezBadillo, J. J. (2013). Training at the optimum power zone produces similar performance improvements to traditional strength training. Journal of Sports Science \& Medicine, 12(1), 109-115.

Maffiuletti, N. A., Dugnani, S., Folz, M., Di Pierno E., \& Mauro, F. (2002). Effect of combined electrostimulation and plyometric training on vertical jump height. Medicine and Science of Sports Exercise, 34(10), 1638-1644.

Maher, C. G., Sherrington, C., Herbert, R. D., Moseley, A. M., \& Elkins, M. (2003). Reliability of the PEDro scale for rating quality of randomized controlled trials. Physical Therapy, 83(8), 713-721.

Malisoux, L., Francaux, M., Nielens, H., \& Theisen, D. (2006). Stretchshortening cycle exercises: an effective training paradigm to enhance power output of human single muscle fibers. Journal of applied physiology, 100(3), 771-779.

Marques, M. A. C., \& Gonzalez-Badillo, J. J. (2005). Oefeito do treino de força sobre o salto vertical em jogadores de basquetebol de 10-13 anos de idade. Revista Brasileira de Ciência e Movimento, 13(2), 715.

Marques, M. C., Tillaar, R. V., Gabbett,T. J., Reis, V. M., \& GonzálezBadillo, J. J. (2009). Physical fitness qualities of professional volleyball players: determination of positional differences. Journal Strength and conditioning Research, 23(4), 1106-1111.

Marques, M. C., Tillaar, R. V., Vescovi, J. D., \& González-Badillo, J. J. (2008). Changes in strength and power performance in elite senior female professional volleyball players during the in-season: a case study. Journal of Strength and Conditining Research, 22(4), 11471155.

Marqueste, T., Messan, F., Hug, F., Laurin, J., Dousset, E., Grelot, L., \& Decheerchi, P. (2010). Effect of Repetitive Biphasic Muscle Electrostimulation Training on Vertical Jump Performances in Female Volleyball Players. International Journal of Sport and Health Science, 8(2010), 50-55

Moher, D., Liberati, A., Tetzlaff, J., Altman, D.G., \& The PRISMA Group (2009). Preferred Reporting Items for Systematic Reviews and Meta-Analyses: The PRISMA Statement. PLoS Medicine, 6(7), e1000097.

Moon, J. R., Eckerson, J. M., Tobkin, S. E., Smith,A. E., Lockwood, C. M., Walter, A.,... Stout, J. R. (2009). Estimating body fat in NCAA Division I female athletes: a five-compartment model validation of laboratory methods. European Journal of Applied Physiology, 105(1), 119-130.

Newton, R. U., Rogers, R. A., Volek, J. S., Häkkinen, K., \& Kraemer, W. J. (2006). Four weeks of optimal load ballistic resistance training at the end of season attenuates declining jump performance of women volleyball players. Journal of Strength and Conditioning Research, 20(4), 955-961.

Pereira, G., Almeida, A. G, Rodacki, A. L. F., Ugrinowitsch, C., Fowler, N.E., \& Kokubun, E. (2008). The influence of resting period length on jumping performance. Journal of Strength and Conditioning Research, 22(4), 1259-1264.

Pereira, A., Costa, A. M., Santos, P., Figueiredo, T., \& João, P. V. (2015). Training strategy of explosive strength in young female volleyball players. Medicina, 51(2), 126-131.

Robinson, L. E., Devor, S. T., Merrick, M. A., \& Buckworth, J. (2004). The effects of land vs. aquatic plyometrics on power, torque, velocity, and muscle soreness in women. Journal of Strength and Conditioning Research, 18(1), 84-91.

Tillman, M. D., Hass, C. J., Brunt, D., \& Bennett, G. R. (2004). Jumping and Landing Techniques in Elite Women's Volleyball. Journal of Sports Science \& Medicine, 3(1), 30-36.

Urrútia, G., y Bonfill, J. (2010). Declaración PRISMA: una propuesta para mejorar la publicación de revisiones sistemáticas y metaanálisis. Medicina Clínica, 135(11), 507-511.

Wilson, G. J., Newton, R. U., Murphy, A. J., \& Humphries, B. J. (1993). The optimal training load for the development of dynamic athletic performance. Medicine and Science in Sports and Exercise, 25(11), 1279-1286

Ziv, G., \& Lidor, R. (2010). Vertical jump in female and male volleyball players: a review of observational and experimental studies. Scandinavian Journal of Medicine \& Science of Sports, 20(4), 556567. 